\title{
Twisted Poisson Homology of Truncated Polynomial Algebras in Four Variables
}

\author{
Yaxiu Wang, Can Zhu, Janhua Hu \\ College of Science, University of Shanghai for Science and Technology, Shanghai, China \\ Email:2471087803@qq.com
}

How to cite this paper: Wang, Y.X., Zhu, C. and Hu, J.H. (2018) Twisted Poisson Homology of Truncated Polynomial Algebras in Four Variables. Journal of Applied Mathematics and Physics, 6, 1817-1824. https://doi.org/10.4236/jamp.2018.69155

Received: August 3, 2018

Accepted: September 8, 2018

Published: September 11, 2018

Copyright ( 2018 by authors and Scientific Research Publishing Inc. This work is licensed under the Creative Commons Attribution International License (CC BY 4.0).

http://creativecommons.org/licenses/by/4.0/

\begin{abstract}
In this paper, we study the twisted Poisson homology of truncated polynomials algebra $A$ in four variables, and we calculate exactly the dimension of $i$-th $(i=1,2,3,4)$ twisted Poisson homology group over $A$ by the induction on the length. The calculation methods provided in this paper can also solve truncated polynomials algebra in a few variables.
\end{abstract}

\section{Keywords}

Twisted Poisson Homology, Poisson Algebra, (Twisted) Poisson Module

\section{Introduction}

For a Poisson algebra, Lichnerowicsz (see [1]) first introduced the notion of Poisson cohomology in 1977. This Poisson cohomology provides important information about the structure of Poisson algebra. Launois S and Richard L (see [2]) studied the Poisson (co)homology of the algebra of the truncated polynomial in two variables and established a duality between the two. Can Zhu (see [3]) proved that this result is still true for all Frobenius Poisson algebra as follows (Theorem 1):

Theorem 1. Let $S$ be a Frobenius Poisson algebra. Then we have the following isomorphism:

$$
H P^{i}(S, S)^{*} \cong H P_{i}\left(S, S_{\sigma}\right),
$$

For all $i \in N$, where $S_{\sigma}$ is the Poisson module induced by the Frobenius isomorphism $\sigma: S \rightarrow S^{*}$ (see [3], Corollary 3.3).

In general, given a Poisson algebra, it is very difficult to calculate its Poisson cohomology. From the above Theorem, the dimension of Poisson cohomology space is determined by calculating twisted Poisson homology. So there is a natu- 
ral problem: how to calculate the twisted Poisson homology of a Poisson algebra. For example, for algebra in [2], how would we calculate its twisted Poisson homology if we extended two variables to four variables or even $n$ variables. The purpose of this paper is to provide a solution to calculate the twisted Poisson homology of truncated polynomials algebra in four variables.

In this article, we will recall some basic knowledge in the second part and show the main conclusions in the third part.

\section{Preliminaries}

Throughout, $\mathbb{k}$ is a field of characteristic zero.

Definition 1 [4]. A right Poisson module $M$ over the Poisson algebra $R$ is a k-vector space $M$ endowed with two bilinear maps - and $\{-,-\}_{M}: M \times R \rightarrow M$ such that

1) $(M, \cdot)$ is a module over the commutative algebra $R$;

2) $\left(M,\{-,-\}_{M}\right)$ is a right Lie-module over the Lie algebra $(R,\{-,-\})$;

3) $\{x a, b\}_{M}=\{x, b\}_{M} a+x\{a, b\}$ for any $a, b \in R$ and $x \in M$;

4) $\{x, a b\}_{M}=\{x, a\}_{M} b+\{x, b\}_{M} a$ for any $a, b \in R$ and $x \in M$;

Left Poisson modules are defined similar. Any Poisson algebra $R$ is naturally a right or left Poisson module over itself.

Definition 2 [5]. Let $A$ be a Poisson algebra. In general, let $\Omega^{1}(A)$ be the Kähler differential module of $A$ and $\Omega^{p}(A):=\wedge^{p} \Omega^{1}(A)$ be the $p$-th Kähler differentia forms, where $\wedge$ is the wedge product over $A$ (also in [6]). Given a right Poisson module $M$ over the Poisson algrbra $A$, there is a canonical chain complex

$$
\begin{aligned}
& \cdots \underset{\partial_{2}}{\longrightarrow} M \otimes_{A} \Omega^{p}(A) \stackrel{\partial_{p}}{\longrightarrow} M \otimes_{A} \Omega^{1} \Omega^{p-1}(A) \stackrel{\partial_{1}}{\longrightarrow} M \otimes_{A} \Omega^{0}(A) \stackrel{\partial_{0-1}}{\longrightarrow} \cdots \\
& \stackrel{\partial_{0}}{\longrightarrow}
\end{aligned}
$$

where for $p \geq 1, \partial_{p}: M \otimes_{A} \Omega^{p}(A) \rightarrow M \otimes_{A} \Omega^{p-1}(A)$ is defined as:

$$
\begin{aligned}
& \partial_{p}\left(m \otimes d a_{1} \wedge \cdots \wedge d a_{p}\right) \\
& =\sum_{i=1}^{p}(-1)^{i-1}\left\{m, a_{i}\right\}_{M} \otimes d a_{1} \wedge \cdots \widehat{d a_{i}} \cdots \wedge d a_{p} \\
& +\sum_{1 \leq i<j \leq p}(-1)^{i+j} m \otimes\left\{a_{i}, a_{j}\right\}_{A} d a_{1} \wedge \cdots \widehat{d a_{i}} \cdots \widehat{d a_{j}} \cdots \wedge d a_{p}
\end{aligned}
$$

The complex (1.1) is called the Poisson complex of $A$ with values in $M$, and for $p \geq 0$ is $p$-th Poisson homology of $A$ with values in $M$, denoed by $H P_{p}(A, M)$.

Definition 3 [5]. Let $D \in \chi^{1}(A)$ be a Poisson derivation, and $M$ be a right Poisson A-module. Define a new bilinear map $\{-,-\}_{M^{D}}: M \times R \rightarrow M$ as

$$
\{m, a\}_{M^{D}}:=\{m, a\}_{M}+m \cdot D(a)
$$

Then the A-module with $\{-,-\}_{M^{D}}$ is a right Poisson A-module, which is called the twisted Poisson module of $M$ twisted by the Poisson derivation $D$, denote by $M^{D}$. 


\section{Twisted Poisson Homology of Truncated Polynomial}

We consider the truncated polynomials algebra

$$
A:=\mathbb{k}\left[x_{1}, x_{2}, x_{3}, x_{4}\right] /\left\langle x_{i} x_{j}-x_{j} x_{i}, x_{i}^{2}\right\rangle
$$

with the Poisson bracket $\left\{x_{i}, x_{j}\right\}_{A}=\lambda_{i j} x_{i} x_{j}, \forall 1 \leq i<j \leq 4, \lambda_{i j} \in \mathbb{k}$. The fact that $\lambda_{i j}=-\lambda_{j i}$ is clear from the definition of Poisson bracket. We can get the modular derivation $D\left(x_{i}\right)=\left(\left(\lambda_{i+1}+\lambda_{i+2}+\cdots+\lambda_{i n}\right)-\left(\lambda_{1 i}+\lambda_{2 i}+\cdots+\lambda_{i-1 i}\right)\right) x_{i}$ in [7]. Then we define a new bilinear map $\{-,-\}_{A^{D}}$ as $\left\{x_{i}, x_{j}\right\}_{A^{D}}:=\left\{x_{i}, x_{j}\right\}_{A}+x_{i} D\left(x_{j}\right)$. By definition 3, $A^{D}$ becomes a twisted Poisson right A-module with $\{-,-\}_{A^{D}}$. Motivated by this result and definition 2, we obtain a new canonical chain complex over $A$ :

$$
\begin{aligned}
& 0 \longrightarrow A^{D} \otimes_{A} \Omega^{4}(A) \stackrel{\delta_{4}^{\pi}}{\longrightarrow} A^{D} \otimes_{A} \Omega^{3}(A) \stackrel{\delta_{3}^{\pi}}{\longrightarrow} A^{D} \otimes_{A} \Omega^{2}(A) \\
& \stackrel{\delta_{2}^{\pi}}{\longrightarrow} A^{D} \otimes_{A} \Omega^{1} \stackrel{\delta_{1}^{\pi}}{\longrightarrow}(A) A^{D} \otimes_{A} \Omega^{0}(A) \stackrel{\delta_{0}^{\pi}}{\longrightarrow} 0
\end{aligned}
$$

where for $1 \leq p \leq 4, \quad \delta_{p}^{\pi}: A^{D} \otimes_{A} \Omega^{p}(A) \rightarrow A^{D} \otimes_{A} \Omega^{p-1}(A)$ is defined as:

$$
\begin{aligned}
& \delta_{p}^{\pi}\left(a_{0} \otimes d a_{1} \wedge \cdots \wedge d a_{p}\right) \\
& =\sum_{i=1}^{p}(-1)^{i-1}\left\{a_{0}, a_{i}\right\}_{A} \otimes d a_{1} \wedge \cdots \widehat{d a}_{i} \cdots \wedge d a_{p} \\
& +\sum_{1 \leq i<j \leq p}(-1)^{i+j} a_{0} \otimes\left\{a_{i}, a_{j}\right\}_{A} d a_{1} \wedge \cdots \widehat{d a_{i}} \cdots \widehat{d a_{j}} \cdots \wedge d a_{p}
\end{aligned}
$$

Now we can get some conclusions as follows.

Proposition 3.1. $H P_{0}\left(A, A^{D}\right)=\mathbb{k}\left(x_{1} x_{2} x_{3} x_{4}\right), \operatorname{dim}\left(H P_{0}\left(A, A^{D}\right)\right)=1$.

Proof. $A^{D} \otimes \Omega^{1}(A) \stackrel{\delta_{1}^{\pi}}{\longrightarrow} A^{D} \otimes \Omega^{0}(A) \stackrel{\delta_{0}^{\pi}}{\longrightarrow} 0$

$$
\delta_{1}^{\pi}: m \otimes d x_{i} \mapsto\left\{m, x_{i}\right\}_{A^{D}}
$$

First of all, we have that $\operatorname{ker} \delta_{0}^{\pi}=A^{D} \otimes \Omega^{0}(A)$, which leads to

$$
H P_{0}\left(A, A^{D}\right)=\operatorname{ker} \delta_{0}^{\pi} / \operatorname{Im} \delta_{1}^{\pi}=A^{D} \otimes \Omega^{0}(A) / \operatorname{Im} \delta_{1}^{\pi},
$$

So we just need to consider which elements in $A^{D} \otimes \Omega^{0}(A)$ have the inverse image.

We proceed by the induction on the length of the elements in $A^{D} \otimes \Omega^{0}(A)$.

Remark: We make an agreement on the length: the length of $1 \otimes d a_{1} \wedge \ldots \wedge d a_{n}$ is 0 ; the length of $a_{1} a_{2} \cdots a_{i} \otimes d a_{j} \wedge \cdots \wedge d a_{n}$ is $i$.

1) The image of element of length 0

$$
\begin{aligned}
1 \otimes d x_{1} & \mapsto\left\{1, x_{1}\right\}_{A^{D}}=D\left(x_{1}\right)=\left(\lambda_{12}+\lambda_{13}+\lambda_{14}\right) x_{1} \\
1 \otimes d x_{2} & \mapsto\left\{1, x_{2}\right\}_{A^{D}}=D\left(x_{2}\right)=\left(\lambda_{23}+\lambda_{24}-\lambda_{12}\right) x_{2} \\
1 \otimes d x_{3} & \mapsto\left\{1, x_{3}\right\}_{A^{D}}=D\left(x_{3}\right)=\left(\lambda_{34}-\lambda_{13}-\lambda_{23}\right) x_{3} \\
1 \otimes d x_{4} & \mapsto\left\{1, x_{4}\right\}_{A^{D}}=D\left(x_{4}\right)=-\left(\lambda_{14}+\lambda_{24}+\lambda_{34}\right) x_{4}
\end{aligned}
$$

Hence, we have: $x_{1}, x_{2}, x_{3}, x_{4} \in \operatorname{Im} \delta_{1}^{\pi}$.

2) The image of element of length 1

$$
x_{1} \otimes d x_{2} \mapsto\left\{x_{1}, x_{2}\right\}_{A} D=\left\{x_{1}, x_{2}\right\}_{A}+x_{1} D\left(x_{2}\right)=\left(\lambda_{23}+\lambda_{24}\right) x_{1} x_{2}
$$




$$
\begin{aligned}
x_{3} \otimes d x_{1} & \mapsto\left\{x_{3}, x_{1}\right\}_{A^{D}}=\left\{x_{3}, x_{1}\right\}_{A}+x_{3} D\left(x_{1}\right)=\left(\lambda_{12}+\lambda_{14}\right) x_{1} x_{3} \\
x_{1} \otimes d x_{4} & \mapsto\left\{x_{1}, x_{4}\right\}_{A^{D}}=\left\{x_{1}, x_{4}\right\}_{A}+x_{1} D\left(x_{4}\right)=-\left(\lambda_{24}+\lambda_{34}\right) x_{1} x_{4} \\
x_{2} \otimes d x_{3} & \mapsto\left\{x_{2}, x_{3}\right\}_{A^{D}}=\left\{x_{2}, x_{3}\right\}_{A}+x_{2} D\left(x_{3}\right)=\left(\lambda_{34}-\lambda_{13}\right) x_{2} x_{3} \\
x_{2} \otimes d x_{4} & \mapsto\left\{x_{2}, x_{4}\right\}_{A^{D}}=\left\{x_{2}, x_{4}\right\}_{A}+x_{2} D\left(x_{4}\right)=-\left(\lambda_{14}+\lambda_{34}\right) x_{2} x_{4} \\
x_{3} \otimes d x_{4} & \mapsto\left\{x_{3}, x_{4}\right\}_{A^{D}}=\left\{x_{3}, x_{4}\right\}_{A}+x_{3} D\left(x_{4}\right)=-\left(\lambda_{14}+\lambda_{24}\right) x_{3} x_{4}
\end{aligned}
$$

Obviously, we get: $x_{i} x_{j} \in \operatorname{Im} \delta_{1}^{\pi}, \forall 1 \leq i<j \leq 4$.

3) The image of element of length 2

$$
\begin{aligned}
x_{1} x_{2} \otimes d x_{3} & \mapsto\left\{x_{1} x_{2}, x_{3}\right\}_{A^{D}}=\left\{x_{1} x_{2}, x_{3}\right\}_{A}+x_{1} x_{2} D\left(x_{3}\right)=\lambda_{34} x_{1} x_{2} x_{3} \\
x_{1} x_{2} \otimes d x_{4} & \mapsto\left\{x_{1} x_{2}, x_{4}\right\}_{A^{D}}=\left\{x_{1} x_{2}, x_{4}\right\}_{A}+x_{1} x_{2} D\left(x_{4}\right)=-\lambda_{34} x_{1} x_{2} x_{4} \\
x_{1} x_{3} \otimes d x_{4} & \mapsto\left\{x_{1} x_{3}, x_{4}\right\}_{A^{D}}=\left\{x_{1} x_{3}, x_{4}\right\}_{A}+x_{1} x_{3} D\left(x_{4}\right)=-\lambda_{24} x_{1} x_{3} x_{4} \\
x_{2} x_{3} \otimes d x_{4} & \mapsto\left\{x_{2} x_{3}, x_{4}\right\}_{A^{D}}=\left\{x_{2} x_{3}, x_{4}\right\}_{A}+x_{2} x_{3} D\left(x_{4}\right)=-\lambda_{14} x_{2} x_{3} x_{4}
\end{aligned}
$$

Hence, we can see: $x_{i} x_{j} x_{k} \in \operatorname{Im} \delta_{1}^{\pi}, \forall 1 \leq i<j<k \leq 4$.

4) The image of element of length 3

$$
\begin{aligned}
& \delta_{1}^{\pi}\left(x_{1} x_{2} x_{3} \otimes d x_{4}\right)=0 \\
& \delta_{1}^{\pi}\left(x_{1} x_{2} x_{4} \otimes d x_{3}\right)=0 \\
& \delta_{1}^{\pi}\left(x_{1} x_{3} x_{4} \otimes d x_{2}\right)=0 \\
& \delta_{1}^{\pi}\left(x_{2} x_{3} x_{4} \otimes d x_{1}\right)=0
\end{aligned}
$$

Hence, $x_{1} x_{2} x_{3} x_{4}$ have no inverse image under the map $\delta_{1}^{\pi}$. Thus $x_{1} x_{2} x_{3} x_{4} \notin \operatorname{Im} \delta_{1}^{\pi}$, and $x_{1} x_{2} x_{3} x_{4} \in H P_{0}\left(A, A^{D}\right)$.

Combined with (1)-(4), it is obvious that

$$
H P_{0}\left(A, A^{D}\right)=\mathbb{k}\left(x_{1} x_{2} x_{3} x_{4}\right), \operatorname{dim}\left(H P_{0}\left(A, A^{D}\right)\right)=1 . \#
$$

Proposition 3.2. $H P_{1}\left(A, A^{D}\right)=\mathbb{k}\left(x_{i} x_{j} x_{k} \otimes d x_{t}\right), 1 \leq i<j<k \leq 4$,

$$
\operatorname{dim}\left(H P_{1}\left(A, A^{D}\right)\right)=4 \text {. }
$$

Proof. $A^{D} \otimes \Omega^{2}(A) \stackrel{\delta_{2}^{\pi}}{\longrightarrow} A^{D} \otimes \Omega^{1}(A) \stackrel{\delta_{1}^{\pi}}{\longrightarrow} A^{D} \otimes \Omega^{0}(A)$

$$
\delta_{1}^{\pi}: m \otimes d x_{i} \mapsto\left\{m, x_{i}\right\}_{A^{D}}
$$

$$
\delta_{2}^{\pi}: m \otimes d x_{i} \wedge d x_{j} \mapsto\left\{m, x_{i}\right\}_{A^{D}} \otimes d x_{j}-\left\{m, x_{j}\right\}_{A^{D}} \otimes d x_{i}-m \otimes d\left\{x_{i}, x_{j}\right\}
$$

In this part, we need to consider two questions: 1) what is the form of the element in $\left.\operatorname{ker} \delta_{1}^{\pi} ; 2\right)$ whether the element in $\operatorname{ker} \delta_{1}^{\pi}$ has the inverse image.

We distinguish four cases below.

1) The element with the length of 0

Since $\delta_{1}^{\pi}\left(1 \otimes d x_{1}\right)=\left\{1, x_{1}\right\}_{A^{D}}=\left(\lambda_{12}+\lambda_{13}+\lambda_{14}\right) x_{1} \neq 0$, we have $1 \otimes d x_{1} \notin \operatorname{ker} \delta_{0}^{\pi}$, and all elements with the form as $1 \otimes d x_{i}$ have the same situation.

2) The element with the length of 1

$$
\delta_{1}^{\pi}\left(C_{12} x_{1} \otimes d x_{2}+C_{21} x_{2} \otimes d x_{1}\right)=\left[C_{12}\left(\lambda_{23}+\lambda_{24}\right)+C_{21}\left(\lambda_{13}+\lambda_{14}\right)\right] x_{1} x_{2}
$$


Let $C_{12} x_{1} \otimes d x_{2}+C_{21} x_{2} \otimes d x_{1} \in \operatorname{ker} \delta_{1}^{\pi}$, then $C_{12}\left(\lambda_{23}+\lambda_{24}\right)+C_{21}\left(\lambda_{13}+\lambda_{14}\right)=0$, we can easy get:

$$
-\left(\lambda_{13}+\lambda_{14}\right) x_{1} \otimes d x_{2}+\left(\lambda_{23}+\lambda_{24}\right) x_{2} \otimes d x_{1} \in \operatorname{ker} \delta_{1}^{\pi}
$$

Similarly, $\quad-\left(\lambda_{12}+\lambda_{14}\right) x_{1} \otimes d x_{3}+\left(\lambda_{32}+\lambda_{34}\right) x_{3} \otimes d x_{1} \in \operatorname{ker} \delta_{1}^{\pi}$

$$
-\left(\lambda_{13}+\lambda_{23}\right) x_{3} \otimes d x_{4}+\left(\lambda_{14}+\lambda_{24}\right) x_{4} \otimes d x_{3} \in \operatorname{ker} \delta_{1}^{\pi}
$$

Now we prove that these elements with the length of 1 in $\operatorname{ker} \delta_{1}^{\pi}$ have inverse image under the map $\delta_{2}^{\pi}$.

$$
\begin{aligned}
\delta_{2}^{\pi}\left(1 \otimes d x_{1} \wedge d x_{2}\right) & =\left\{1, x_{1}\right\}_{A^{D}} \otimes d x_{2}-\left\{1, x_{2}\right\}_{A^{D}} \otimes d x_{1}-1 \otimes d\left\{x_{1}, x_{2}\right\} \\
& =D\left(x_{1}\right) \otimes d x_{2}-D\left(x_{2}\right) \otimes d x_{1}-1 \otimes d\left\{x_{1}, x_{2}\right\} \\
& =\left(\lambda_{13}+\lambda_{14}\right) x_{1} \otimes d x_{2}-\left(\lambda_{23}+\lambda_{24}\right) x_{2} \otimes d x_{1} \\
\delta_{2}^{\pi}\left(1 \otimes d x_{1} \wedge d x_{3}\right) & =\left\{1, x_{1}\right\}_{A^{D}} \otimes d x_{3}-\left\{1, x_{3}\right\}_{A^{D}} \otimes d x_{1}-1 \otimes d\left\{x_{1}, x_{3}\right\} \\
& =D\left(x_{1}\right) \otimes d x_{3}-D\left(x_{3}\right) \otimes d x_{1}-1 \otimes d\left\{x_{1}, x_{3}\right\} \\
& =\left(\lambda_{12}+\lambda_{14}\right) x_{1} \otimes d x_{2}-\left(\lambda_{32}+\lambda_{34}\right) x_{3} \otimes d x_{1} \\
& \quad \vdots \\
\delta_{2}^{\pi}\left(1 \otimes d x_{3} \wedge d x_{4}\right) & =\left\{1, x_{3}\right\}_{A^{D}} \otimes d x_{4}-\left\{1, x_{4}\right\}_{A^{D}} \otimes d x_{3}-1 \otimes d\left\{x_{3}, x_{4}\right\} \\
& =D\left(x_{3}\right) \otimes d x_{4}-D\left(x_{4}\right) \otimes d x_{3}-1 \otimes d\left\{x_{3}, x_{4}\right\} \\
& =-\left(\lambda_{13}+\lambda_{23}\right) x_{3} \otimes d x_{4}+\left(\lambda_{14}+\lambda_{24}\right) x_{4} \otimes d x_{3}
\end{aligned}
$$

3) The element with the length of 2

$$
\begin{aligned}
& \delta_{1}^{\pi}\left(C_{123} x_{1} x_{2} \otimes d x_{3}+C_{132} x_{1} x_{3} \otimes d x_{2}+C_{231} x_{2} x_{3} \otimes d x_{1}\right) \\
& =\left(C_{123} \lambda_{34}+C_{132} \lambda_{24}+C_{231} \lambda_{14}\right) x_{1} x_{2} x_{3}
\end{aligned}
$$

If $C_{123} \lambda_{34}+C_{132} \lambda_{24}+C_{231} \lambda_{14}=0 \quad$ (1.2), so that

$$
C_{123} x_{1} x_{2} \otimes d x_{3}+C_{132} x_{1} x_{3} \otimes d x_{2}+C_{231} x_{2} x_{3} \otimes d x_{1} \in \operatorname{ker} \delta_{1}^{\pi}
$$

For (1.2), let $C_{123}$ be a free variable, we can infer that

$$
\left\{\begin{array} { l } 
{ C _ { 1 2 3 } \lambda _ { 3 4 } + C _ { 1 3 2 } \lambda _ { 2 4 } = 0 } \\
{ C _ { 1 2 3 } \lambda _ { 3 4 } + C _ { 2 3 1 } \lambda _ { 1 4 } = 0 }
\end{array} \Rightarrow \left\{\begin{array} { l } 
{ C _ { 1 3 2 } = \frac { - C _ { 1 2 3 } \lambda _ { 3 4 } } { \lambda _ { 2 4 } } } \\
{ C _ { 2 3 1 } = \frac { - C _ { 1 2 3 } \lambda _ { 3 4 } } { \lambda _ { 1 4 } } }
\end{array} \Rightarrow \left\{\begin{array}{l}
x_{1} x_{2} \otimes d x_{3}-\frac{\lambda_{34}}{\lambda_{24}} x_{1} x_{3} \otimes d x_{2} \in \operatorname{ker} \delta_{1}^{\pi} \\
x_{1} x_{2} \otimes d x_{3}-\frac{\lambda_{34}}{\lambda_{14}} x_{2} x_{3} \otimes d x_{1} \in \operatorname{ker} \delta_{1}^{\pi}
\end{array}\right.\right.\right.
$$

similarly,

$$
\begin{aligned}
& x_{1} x_{3} \otimes d x_{4}-\frac{\lambda_{24}}{\lambda_{23}} x_{1} x_{4} \otimes d x_{3} \in \operatorname{ker} \delta_{1}^{\pi} \\
& x_{1} x_{3} \otimes d x_{4}+\frac{\lambda_{24}}{\lambda_{12}} x_{3} x_{4} \otimes d x_{1} \in \operatorname{ker} \delta_{1}^{\pi}
\end{aligned}
$$

We can find the inverse image of all elements as the above by following 


$$
\begin{aligned}
& \delta_{2}^{\pi}\left(\frac{1}{\lambda_{24}} x_{1} \otimes d x_{2} \wedge d x_{3}\right)=x_{1} x_{2} \otimes d x_{3}-\frac{\lambda_{34}}{\lambda_{24}} x_{1} x_{3} \otimes d x_{2} \\
& \delta_{2}^{\pi}\left(\frac{1}{\lambda_{14}} x_{2} \otimes d x_{1} \wedge d x_{3}\right)=x_{1} x_{2} \otimes d x_{3}-\frac{\lambda_{34}}{\lambda_{14}} x_{2} x_{3} \otimes d x_{1} \\
& \delta_{2}^{\pi}\left(-\frac{1}{\lambda_{23}} x_{1} \otimes d x_{3} \wedge d x_{4}\right)=x_{1} x_{3} \otimes d x_{4}-\frac{\lambda_{24}}{\lambda_{23}} x_{1} x_{4} \otimes d x_{3} \\
& \delta_{2}^{\pi}\left(\frac{1}{\lambda_{12}} x_{3} \otimes d x_{1} \wedge d x_{4}\right)=x_{1} x_{3} \otimes d x_{4}+\frac{\lambda_{24}}{\lambda_{12}} x_{3} x_{4} \otimes d x_{1}
\end{aligned}
$$

4) The element with the length of 3

For $\forall 1 \leq i<j<k \leq 4, \delta_{1}^{\pi}\left(x_{i} x_{j} x_{k} \otimes d x_{t}\right)=0$, indeed, for $\forall 1 \leq i<j \leq 4,1 \leq k<t \leq 4, \delta_{2}^{\pi}\left(x_{i} x_{j} \otimes d x_{k} \wedge d x_{t}\right)=0$. Since the map $\delta_{p}^{\pi}$ keeps the variable unchanged, so we can't find the inverse image of $x_{i} x_{j} x_{k} \otimes d x_{t}$ under the map $\delta_{2}^{\pi}$.

In conclusion, only $x_{i} x_{j} x_{k} \otimes d x_{t} \in H P_{1}\left(A, A^{D}\right)$, and $\operatorname{dim}\left(H P_{0}\left(A, A^{D}\right)\right)=C_{4}^{1}=4$.

Similar to the proof of proposition 3.2, we can prove the following

Proposition 3.3. $H P_{2}\left(A, A^{D}\right)=\mathbb{k}\left(x_{i} x_{j} \otimes d x_{k} \wedge d x_{t}\right),(1 \leq i<j \leq 4,1 \leq k<t \leq 4)$ $\operatorname{dim}\left(H P_{2}\left(A, A^{D}\right)\right)=6$.

Proof. $A^{D} \otimes \Omega^{3}(A) \stackrel{\delta_{3}^{\pi}}{\longrightarrow} A^{D} \otimes \Omega^{2}(A) \stackrel{\delta_{2}^{\pi}}{\longrightarrow} A^{D} \otimes \Omega^{1}(A)$

$$
\begin{array}{r}
\delta_{2}^{\pi}: m \otimes d x_{i} \wedge d x_{j} \mapsto\left\{m, x_{i}\right\}_{A^{D}} \otimes d x_{j}-\left\{m, x_{j}\right\}_{A^{D}} \otimes d x_{i}-m \otimes d\left\{x_{i}, x_{j}\right\} \\
\delta_{3}^{\pi}: m \otimes d x_{i} \wedge d x_{j} \wedge d x_{k} \mapsto\left\{m, x_{i}\right\}_{A^{D}} \otimes d x_{j} \wedge d x_{k}-\left\{m, x_{j}\right\}_{A^{D}} \otimes d x_{i} \wedge d x_{k} \\
+\left\{m, x_{k}\right\}_{A^{D}} \otimes d x_{i} \wedge d x_{j}-m \otimes d\left\{x_{i}, x_{j}\right\} \wedge d x_{k} \\
+m \otimes d\left\{x_{i}, x_{k}\right\} \wedge d x_{j}-m \otimes d\left\{x_{j}, x_{k}\right\} \wedge d x_{i}
\end{array}
$$

1) The element with the length of 0

When we calculate the 1-th twisted Poisson homology group, we have found that each element of length 0 in $A^{D} \otimes \Omega^{2}(A)$ has an image under the map $\delta_{2}^{\pi}$, and never belongs to $\operatorname{ker} \delta_{2}^{\pi}$.

2) The element with the length of 1

$$
\begin{aligned}
& \delta_{2}^{\pi}\left(k_{1} x_{1} \otimes d x_{3} \wedge d x_{4}+k_{3} x_{3} \otimes d x_{1} \wedge d x_{4}+k_{4} x_{4} \otimes d x_{1} \wedge d x_{3}\right) \\
& =\left(k_{3} \lambda_{12}-k_{1} \lambda_{23}\right) x_{1} x_{3} \otimes d x_{4}+\left(k_{1} \lambda_{24}+k_{4} \lambda_{12}\right) x_{1} x_{4} \otimes d x_{3}+\left(k_{3} \lambda_{24}+k_{4} \lambda_{23}\right) x_{3} x_{4} \otimes d x_{1}
\end{aligned}
$$

$x_{1} x_{3} \otimes d x_{4}, \quad x_{1} x_{4} \otimes d x_{3}$ and $x_{3} x_{4} \otimes d x_{1}$ are linear independence. If and only if such that $k_{3} \lambda_{12}-k_{1} \lambda_{23}=0, k_{1} \lambda_{24}+k_{4} \lambda_{12}=0$ and $k_{3} \lambda_{24}+k_{4} \lambda_{23}=0$ at same time, i.e.,

$$
k_{3}=\frac{k_{1} \lambda_{23}}{\lambda_{12}}, k_{4}=-\frac{k_{1} \lambda_{24}}{\lambda_{12}},
$$

we get that $x_{1} \otimes d x_{3} \wedge d x_{4}+\frac{k_{1} \lambda_{23}}{\lambda_{12}} x_{3} \otimes d x_{1} \wedge d x_{4}-\frac{k_{1} \lambda_{24}}{\lambda_{12}} x_{4} \otimes d x_{1} \wedge d x_{3} \in \operatorname{ker} \delta_{2}^{\pi}$. 
Similarly,

$\delta_{3}^{\pi}\left(\frac{1}{\lambda_{12}} \otimes d x_{1} \wedge d x_{3} \wedge d x_{4}\right)=x_{1} \otimes d x_{3} \wedge d x_{4}+\frac{k_{1} \lambda_{23}}{\lambda_{12}} x_{3} \otimes d x_{1} \wedge d x_{4}-\frac{k_{1} \lambda_{24}}{\lambda_{12}} x_{4} \otimes d x_{1} \wedge d x_{3}$

Obviously, the preimage of

$x_{1} \otimes d x_{3} \wedge d x_{4}+\frac{k_{1} \lambda_{23}}{\lambda_{12}} x_{3} \otimes d x_{1} \wedge d x_{4}-\frac{k_{1} \lambda_{24}}{\lambda_{12}} x_{4} \otimes d x_{1} \wedge d x_{3}$

$\frac{1}{\lambda_{12}} \otimes d x_{1} \wedge d x_{3} \wedge d x_{4}$. Thus, this element in $\operatorname{ker} \delta_{2}^{\pi}$ does not belong to $H P_{2}\left(A, A^{D}\right)$. It is clear that the element with the same form have the same situation.

3) The element with the length of 2

For $1 \leq i<j \leq 4,1 \leq k<t \leq 4, \quad \delta_{2}^{\pi}\left(x_{i} x_{j} \otimes d x_{k} \wedge d x_{t}\right)=0$. Also $\delta_{3}^{\pi}\left(x_{i} \otimes d x_{j} \wedge d x_{k} \wedge d x_{t}\right)=0$.

This means that, the element $x_{i} x_{j} \otimes d x_{k} \wedge d x_{t}$ has no inverse image under the map $\delta_{3}^{\pi}$.

Thus, only $x_{i} x_{j} \otimes d x_{k} \wedge d x_{t} \in H P_{2}\left(A, A^{D}\right), \quad(1 \leq i<j \leq 4,1 \leq k<t \leq 4)$.

In conclusion, it suffices to show that $H P_{2}\left(A, A^{D}\right)=\mathbb{k}\left(x_{i} x_{j} \otimes d x_{k} \wedge d x_{t}\right)$, moreover

$$
\operatorname{dim}\left(H P_{2}\left(A, A^{D}\right)\right)=C_{4}^{2}=6
$$

Proposition 3.4. $\operatorname{dim}\left(H P_{3}\left(A, A^{D}\right)\right)=3, \operatorname{dim}\left(H P_{4}\left(A, A^{D}\right)\right)=0$.

Proof. $0 \longrightarrow A^{D} \otimes \Omega^{4}(A) \stackrel{\delta_{4}^{\pi}}{\longrightarrow} A^{D} \otimes \Omega^{3}(A) \stackrel{\delta_{3}^{\pi}}{\longrightarrow} A^{D} \otimes \Omega^{2}(A)$

$$
\begin{aligned}
\delta_{4}^{\pi}\left(1 \otimes d x_{1} \wedge d x_{2} \wedge d x_{3} \wedge d x_{4}\right)= & \left(-\lambda_{12}-\lambda_{14}-\lambda_{13}\right) x_{1} \otimes d x_{2} \wedge d x_{3} \wedge d x_{4} \\
& +\left(\lambda_{21}+\lambda_{23}+\lambda_{24}\right) x_{2} \otimes d x_{1} \wedge d x_{3} \wedge d x_{4} \\
& +\left(\lambda_{13}+\lambda_{23}+\lambda_{43}\right) x_{3} \otimes d x_{1} \wedge d x_{2} \wedge d x_{4} \\
& +\left(-\lambda_{14}-\lambda_{34}-\lambda_{24}\right) x_{4} \otimes d x_{1} \wedge d x_{2} \wedge d x_{3} \\
& :=t_{1} X_{1}+t_{2} X_{2}+t_{3} X_{3}+t_{4} X_{4}
\end{aligned}
$$

When we calculate the 2-th twisted Poisson homology group, we have noticed that each element like $1 \otimes d x_{i} \wedge d x_{j} \wedge d x_{k} \quad$ always has a image under the map $\delta_{3}^{\pi}$, that means $1 \otimes d x_{i} \wedge d x_{j} \wedge d x_{k} \notin \operatorname{ker} \delta_{3}^{\pi}$. On the other hand, $\delta_{3}^{\pi}\left(x_{i} \otimes d x_{j} \wedge d x_{k} \wedge d x_{t}\right)=0$, implies that, $\operatorname{dim}\left(\operatorname{ker} \delta_{3}^{\pi}\right)=4$, indeed, $\operatorname{dim}\left(\operatorname{Im} \delta_{4}^{\pi}\right)=1$, so that $\operatorname{dim}\left(H P_{3}\left(A, A^{D}\right)\right)=3$.

Obviously, since $H P_{4}\left(A, A^{D}\right)=0$, we have $\operatorname{dim}\left(H P_{4}\left(A, A^{D}\right)\right)=0$.

\section{Conflicts of Interest}

The authors declare no conflicts of interest regarding the publication of this paper.

\section{References}

[1] Lichnerowicz, A. (1977) Les variétés de Poisson et leurs algèbres de Lie associées, (French). Journal of Differential Geometry, 12, 253-300. 
https://doi.org/10.4310/jdg/1214433987

[2] Launois, S. and Richard, L. (2007) Twisted Poincare Duality for Some Quadratic Poisson Algebras. Letters in Mathematical Physics, 79, 161-174.

https://doi.org/10.1007/s11005-006-0133-Z

[3] Zhu, C., Van Oystaeyen, F. and Zhang, Y. (2014) On (Co)homology of Frobenius Poisson Algebras. Journal of K-Theory. K-Theory and Its Applications to Algebra, Geometry, and Topology, 14, 371-386. https://doi.org/10.1017/is014007026jkt276

[4] Oh, S.Q. (2007) Poisson Enveloping Algebras. Communications in Algebra, No. 27, 2181-2186.

[5] Luo, J., Wang, S.Q. and Wu, Q.S. (2015) Twisted Poincaré Duality between Poisson Homology and Poisson Cohomology. Journal of Algebra, 442, 484-505. https://doi.org/10.1016/j.jalgebra.2014.08.023

[6] Oh, S.Q. (2006) Poisson Polynomial Rings. Communications in Algebra, 34, 1265-1277. https://doi.org/10.1080/00927870500454463

[7] Wang, M.Y. (2018) Poisson (Co)homology of a Class of Frobenius Poisson Algebras. Journal of Applied Mathematics and Physics, 6, 530-553.

https://doi.org/10.4236/jamp.2018.63048 